\title{
Fuzzy Logic Controlled Based Ant-Lion Optimization Hybridization for Economic Power Dispatch
}

\author{
Mercy Rosalina Kotapuri*, Rajesh Kumar Samala \\ Department of Electrical and Electronics Engineering, VFSTR (Deemed to be University), Vadlamudi, Guntur 522213, India
}

Corresponding Author Email: kmr_eee@vignan.ac.in

https://doi.org/10.18280/jesa.530515

Received: 20 April 2020

Accepted: 14 August 2020

\section{Keywords:}

economic dispatch, ant-lion optimization, fuzzy logic controller, fuel cost

\begin{abstract}
The demand on the power system rising more rapidly is causing to increase the power system size and capacity. There is a need of interconnection of various generating stations to meet the increased load demand. Economical unit commitment is necessary for plant operation with the advancement in power system integration. The Economical Power Dispatch (EPD) is to find the most favourable combination of generating systems output powers which reduce the fuel cost by satisfying all system constraints. This research involves the fuzzy logic controller (FLC) has been hybridized with Ant-Lion Optimization (ALO) algorithm for EPD. By using this new hybrid technique, minimization of total operating cost by economically dispatch the power to meet the required load and also minimization of system total losses by optimum allocation of DG units were done. Fuel cost function and demand on system are modeled by fuzzy membership functions. The ALO is used to obtain the schedule the committed generating unit's outputs so as to meet the required load demand. This proposed FLC based ALO technique executed with MATLAB software and applied on IEEE-30 system. Effectiveness of this projected algorithm is determined and evaluated with standalone techniques like conventional ALO, ALO-PSO algorithms.
\end{abstract}

\section{INTRODUCTION}

The In electric power system it is always very important to find the effective and optimal economical operation. In recent years, to make revenue, it becomes much essential for all utilities run their systems with lowest cost by satisfying customer demand. With the huge increasing demand on the power system and the minimum generating systems availability, and the limitations of supply and the cost of fuel, all the committed units should produce the energy to the demand with the minimum fuel cost.

The main purpose of EPD is to operate and produce the committed unit's power outputs, to meet the required load at low operating cost while satisfying all system constraints [1]. Many researchers have undertaken the EPD problem [2]. The EPD issue involved many different issues like pre-dispatch issue or unit commitment i.e. it is essential to find the best units from the all available units and operate to meet the variable load and other is EPD is economic power dispatch, in which it is necessary to dispatch the load among the committed units in such a way that the total operating cost should be minimum.

In EPD, the energy delivered by committed units is not constant. It is allowed to produce within particular limits to meet particular demand with low consumption of fuel.

For protection of environment based on rules from government, the traditional way of dispatching electrical energy at low cost is not only the solution. The minimization of pollution is also a considerable factor [3]. In order to reduce the level of $\mathrm{NO}$ and $\mathrm{SO}_{2}$, which are delivered from different sources, likes preparing a cleaning scheme for postcombustion. Development of eco-friendly economical dispatch is advisable for the system operation due to ease of apply and need low extra cost. IEEE present operating issues working sets reported regulations for clean environment effect on network operation. Various techniques were projected like Gent [4] in early, provided an economical dispatch approach in 1970 with traditional technique of optimization. Very recently an artificial intelligence [5] and genetic algorithm [6, 7] were applied on the test system to solve this EPD issue. An artificial intelligence-based GA (Genetic Algorithm) has projected themselves as an efficient technique for optimization. In this approach, genetic operators like mutation and crossover has substantial effect on performance.

There has been an increased interest in installing DG at the distribution schemes because of considerable compensations like power loss reduction, cost reduction, environmental friendliness, voltage enhancement, postponement of system upgrades and amassed reliability [8,9]. Decision about DG placement is taken by their owners and investors, depending on site and primary fuel availability or climatic conditions. The placement should be optimal in order, for maximum user benefit and minimum congestion of DG applied in the network $[10,11]$. Although the installation and manipulation of Depending on the number of DGs to be installed, the ODGP issue is categorized as: 1) single DG or 2) multiple DGs installation. Numerous approaches have been described for optimal sitting and sizing of the DG through dissimilar optimization method enlightening technical and economical performances [12-15]. Tools such as deterministic, heuristic and hybrid methods are promising and still sprouting in this field. In addition, abridging assumptions have been utilized to augment the solution performance. Besides power loss 
reduction, locating a DG may be on the basis of cost reduction. Predictably, the mixed integer linear program, Genetic Algorithm (GA), Tabu Search (TS), Particle Swarm Optimization (PSO) algorithm, direct search algorithm and Ant Colony Optimization (ACO) algorithm are used to investigate the optimal placement and sizing of DG. In those articles while placing the DGs emphasis is provided on the reduction of line loss mostly. Some of those have tried to progress the system voltage also [16-18]. Consequently, the optimization methods should be engaged for deregulation of power industry, permitting for the best apportionment of the DG. In the article, an effective algorithm is proposed to scrutinize the load flow issue and the placement problem of DG.

In the article, an effective algorithm is proposed to scrutinize the load flow issue and the placement problem of DG to minimize test system total losses and economic dispatch of power by optimal scheduling of committed generating units to reduce the total operating cost by satisfying all system constraints.

\section{A BRIEF REVIEW: RECENT RESEARCH WORK}

Many conventional and latest methods had been used for solving the economic power dispatch issues by considering various objective functions. Different conventional techniques like lambda iterative technique, gradient-based technique, Bundle technique [19], non-linear method [20], mixed integer liner programming method [21], Newton-based method [22] etc., were present in literature for solving this economic power dispatch problem. But these existing methods have some disadvantages and failed to solve economic power dispatch issue. Along with these some population based meta-heuristic methods in literature have proved themselves effective performance. Such as Tabu search [23], Genetic Algorithm [24], Ant-Colony Optimization [25], Particle Swarm Optimization [26], Gravitational Search Algorithm with FireFly [27] etc. These methods also always not guarantee in finding optimal outcome. These heuristic methods are also having their disadvantages slow rate of convergence around global solution, time consuming is high, not easy to tune control parameters, difficult in produce effective memory system, trapping into local optima. ALO technique imitates the catching behaviour of ant lions in general. The updating process is done with the elitism phase of ALO. The proposed hybrid technique improves the ability of global search of objective function which provides the better opportunity to increase the solution space. This also provides the global solution for EPD problem. The effectiveness of proposed technique was evaluated with IEEE-30 sus system with six generators in the system. The outcomes obtained by this proposed method compared with conventional ALO and ALO-PSO methods.

New genetic method based on arithmetic crossover was presented to improve the ELD solution quality. In this research arithmetic crossover, elitism both defines a linear combination of two chromosomes and to produce possible operating consecutive sets mutation used in the GA [28].

The solution for ELD problem was presented with line flow constraints the genetic algorithm application. The major advantage of this GA is that it used only the pay of information; hence it is nature independent of search space like smoothness and convexity [29].
An approach using fuzzy decision tree was presented for ELD problem. In this research an improvement has done in DT approach by fuzzy logic addition to load and unit limits. With this there is an improvement in the convergence rate. The cost of generation obtained is also less than the traditional formulation [30].

One hybrid algorithm was presented to solve the ELD problem is the genetic algorithm with fuzzy logic controller with multi-shunt flexible AC transmission systems, which continuously adjust parameters of mutation and crossover [31]

One new formulation was presented for economic dispatch to solve economic load dispatch problem. By reducing the variable numbers and by removing constraints of equality and inequality the new formulation has done. This transformed the constrained non-linear programming into unconstrained one. This new unconstrained formulation objective function is reduced by Hooke-Jeeves' approach [32].

An effective optimization approach was presented to solve ELD problem based on genetic algorithm with continuous and non-smooth cost function by considering various constraints. The presented algorithm effectiveness finds out on various systems with system losses of thermal power plant [33].

Four evolutionary algorithms study was presented for ELD problem solvation, they are particle swarm optimization, genetic algorithm, ant-colony optimization and bacteria foraging optimization. This research is carried on IEEE-30 test system [34].

\section{MATHEMATICAL FORMULATION FOR} OBJECTIVE FUNCTION

The purpose of the economic dispatch is to schedule the outputs of all available generation units in the power system such that the fuel cost is minimized while system constraints are satisfied.

The objective function of an EPD problem is to:

$$
\min \text { imize } F_{\cos t}=\sum_{j=1}^{N_{g}} F_{j}\left(P_{j}\right)
$$

The fitness function for the selected objective function is:

$$
F_{j}\left(P_{j}\right)=a_{j}+b_{j} P_{j}+c_{j} P_{j}^{2}
$$

$a_{j}, b_{j}$ and $c_{j}$ represent the cost coefficients of the $j^{\text {th }}$ generating unit.

$F_{j}\left(P_{j}\right)$ represents the cost function of the $j^{\text {th }}$ generating units (in $\$ / \mathrm{h}$ ).

$P_{j}$ represents the real output of the $j^{\text {th }}$ generating units (in $\mathrm{MW}$ ).

$N_{g}$ is the total number of generators in the power system.

Subjected to the following generator constraints:

$$
\sum_{j=1}^{N_{g}} P_{j}=P_{D}+P_{L}
$$

\section{PROPOSED EFFICIENT TECHNIQUE FOR ECONOMICAL POWER DISPATCH}

In this research, the one hybridization method is presented 
to solve the EPD issue. To analyse the generating unit's outputs from the committed generating units, the objective function is defined for IEEE-30 mesh configuration network. By using this new hybrid technique, minimization of cost function by economically dispatch the power to meet the required load and also minimization of system total losses. For this, the test system bus data, line data, committed generating unit's capacities, minimum and maximum generation capacities, are considered as the input for this new proposed hybrid approach. The total working procedure this new proposed hybrid approach for EPD is demonstrated in Figure 1.

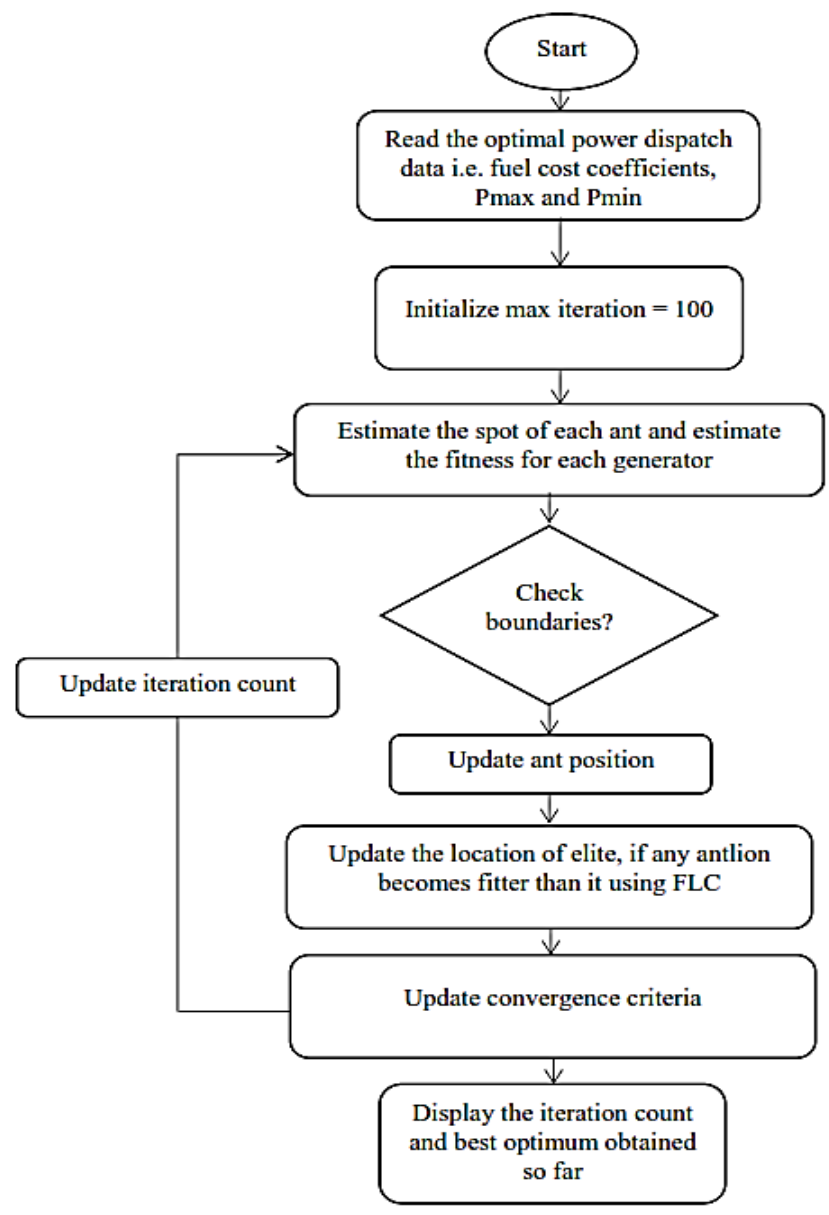

Figure 1. New hybrid technique flow chart

\subsection{Fuzzy logic controller using Mamdani}

For this new hybrid approach using Fuzzy Logic controller for inference the system using Mamdani type. The Loss sensitivity Factor (LSF) and the node voltages are considered as the input to the FLC and the fitness evaluation is considered as the output of the FLC.

Triangle membership functions are considered to construct the membership function plots.

Figure 2 shows that the node voltage triangle membership function plot and the low, medium and high are considered as membership function and this is ranging from $0-1$.

Figure 3 shows that the LSF triangle membership function plot and the low, medium and high are considered as membership function and this is ranging from $0.9-1.1$.

Figure 4 shows that the fitness triangle membership function plot and the low, medium and high are considered as membership function and this is ranging from $0-1$.

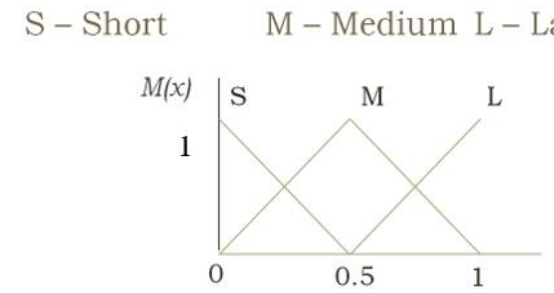

Figure 2. Membership function plot for p.u. Nodal Voltage

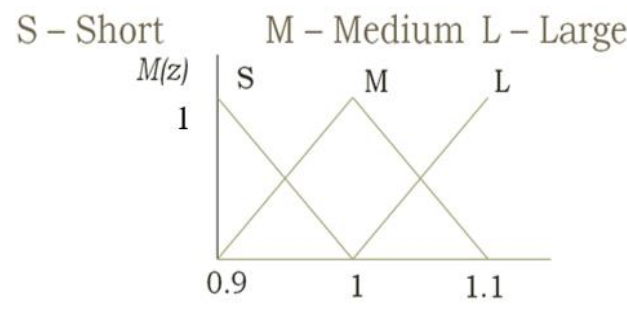

Figure 3. Membership function plot for Loss Sensitivity Factor (LSF)

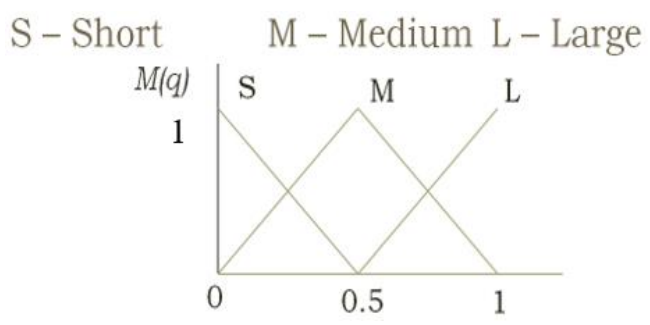

Figure 4. Membership function for fitness

Table 1 represents the rule base for fitness.

Table 1. Decision matrix for determining fitness rule base

\begin{tabular}{cccc}
\hline LSF\Voltage & S & M & L \\
\hline S & S & S & M \\
M & S & M & M \\
L & M & M & L \\
\hline
\end{tabular}

\section{The ALO for training dataset}

The ALO approach is used for optimal training dataset is briefly described by following section.

\section{The random placements of the ant's and ant-Lion's}

The random location of the ant's and Ant-Lion's are represented in $\mathbf{M}_{\text {ant-lion, }} \mathbf{M}_{\text {ant }}$ [35].

$$
\begin{gathered}
M_{\text {ant-lion }}=\left(\begin{array}{cccc}
A L_{1,1} & A L_{1,2} & \ldots & A L_{1, n} \\
A L_{2,1} & A L_{2,2} & \ldots & A L_{2, d} \\
\vdots & \vdots & \vdots & \vdots \\
A L_{n, 1} & A L_{n, 2} & \ldots & A L_{n, d}
\end{array}\right) \\
M_{\text {ant }}=\left(\begin{array}{cccc}
A_{1,1} & A_{1,2} & \ldots & A_{1, n} \\
A_{2,1} & A_{2,2} & \ldots & A_{2, d} \\
\vdots & \vdots & \vdots & \vdots \\
A_{n, 1} & A_{n, 2} & \ldots & A_{n, d}
\end{array}\right)
\end{gathered}
$$

Fitness function of all ant-lion's and ant's has been given in the matrix $\mathrm{M}_{\mathrm{OAL}}, \mathrm{M}_{\mathrm{OA}}[36]$ and function $\mathrm{f}$ is given as, 


$$
\begin{gathered}
M_{O A L}=\left(\begin{array}{c}
f\left(\left[A L_{1,1}, A L_{1,2}, \ldots, A L_{1, n}\right]\right) \\
f\left(\left[A L_{2,1}, A L_{2,2}, \ldots, A L_{2, d}\right]\right) \\
\vdots \\
f\left(\left[A L_{n, 1}, A L_{n, 2}, \ldots, A L_{n, d}\right]\right)
\end{array}\right) \\
M_{O A}=\left(\begin{array}{c}
f\left(\left[A_{1,1}, A_{1,2}, \ldots, A_{1, n}\right]\right) \\
f\left(\left[A_{2,1}, A_{2,2}, \ldots, A_{2, d}\right]\right) \\
\vdots \\
f\left(\left[A_{n, 1}, A_{n, 2}, \ldots, A_{n, d}\right]\right)
\end{array}\right)
\end{gathered}
$$

\section{Ant's Movement randomly}

For search of pray total ants permitted to move randomly. The movement of ant's is shown by (7),

$$
X(t)=\left[\begin{array}{l}
0, \operatorname{cumsum}\left(2 r\left(t_{1}\right)-1\right), \operatorname{cumsum}\left(2 r\left(t_{2}\right)-1\right), \\
\ldots, \operatorname{cumsum}\left(2 r\left(t_{n}\right)-1\right)
\end{array}\right]
$$

where, cumsum represents the total sum, $n$ represents total ants, $t$ represents step random walk, and random generation represents with $r(t)$, production of random number between $[0,1]$ with uniform distribution,

$$
r(t)=\left\{\begin{array}{l}
1 \text { if rand }>0.5 \\
0 \text { if rand } \leq 0.5
\end{array}\right.
$$

Updating the position of ant's as,

$$
X_{i}^{t}=\frac{\left(X_{i}^{t}-p_{i}\right)\left(s_{i}-r_{i}^{t}\right)}{s_{i}^{t}-p_{i}}+r_{t}
$$

Here, $q_{i}$ and $p_{i}$ are max and min values of ant's walk randomly, $r_{i}^{t}$ and $s_{i}^{t}$ signifies min and max $m^{\text {th }}$ variable at $t^{\text {th }}$ iteration.

\section{Ants trapping}

Random walk of ant's is changed by traps, which are developed by ant lion's given by:

$$
\begin{gathered}
c_{m}^{t}=A n t-\operatorname{lion}_{n}^{t}+c^{t} \\
d_{m}^{t}=A n t-\operatorname{lion}_{n}^{t}+d^{t}
\end{gathered}
$$

\section{Trap construction}

The ALO algorithm is for ant-lion's selection by roulette wheel operator depends on ant-lion fitness.

\section{Ants sliding into pits}

Sliding of ant's into traps characterized by,

$$
\begin{aligned}
& c^{t}=\frac{c^{t}}{I} \\
& d^{t}=\frac{d^{t}}{I}
\end{aligned}
$$

Here, $I=10^{\mathrm{w} t / \mathrm{s}}, t$ is current iteration, $S$ is the max iterations and $\mathrm{w}$ is a constant, and its value is given by,

$$
w= \begin{cases}2 & \text { if } t>0.1 S \\ 3 & \text { if } t>0.5 S \\ 4 & \text { if } t>0.75 S \\ 5 & \text { if } t>0.9 S \\ 6 & \text { if } t>0.95 S\end{cases}
$$

\section{Prey catching and pit reconstruction}

To catch the new ants, now ant-lions update their current location,

$$
\text { Ant }- \text { lion }_{n}^{t}=A n t_{m}^{t} \quad \text { if } f\left(A n t_{m}^{t}\right)>f\left(\text { Ant }- \text { lion }_{j}^{t}\right)
$$

\section{Elitism}

Every ant is estimated to associate with an ant-lion using roulette wheel as,

$$
A n t^{t}=\frac{R_{A}^{t}+R_{B}^{t}}{2}
$$

\section{OUTCOMES AND DISCUSSIONS}

In this section of the paper, the entire work is explained in a precise manner. The standard IEEE-30 bus system as in Figure 5 [37] is used as a test system and the results are evaluated. The test system consists of 30 buses of which 6 generating buses are present including slack bus and remaining 24 are load buses. 41 lines, 4 tap changing transformers, and 9 shunt capacitors are present in this test case. MATLAB software is used to run NR power flow.

In this research, All the outcomes using various methods of algorithms are reported in Tables 2 to 4. The Comparison Analysis using various methods illustrated in Figures 6 to 8 . This proposed FLC hybrid method has been tested for solving IEEE-30 bus. The response of proposed FLC hybrid method is also evaluated with outcomes of ALO and ALO-PSO techniques.

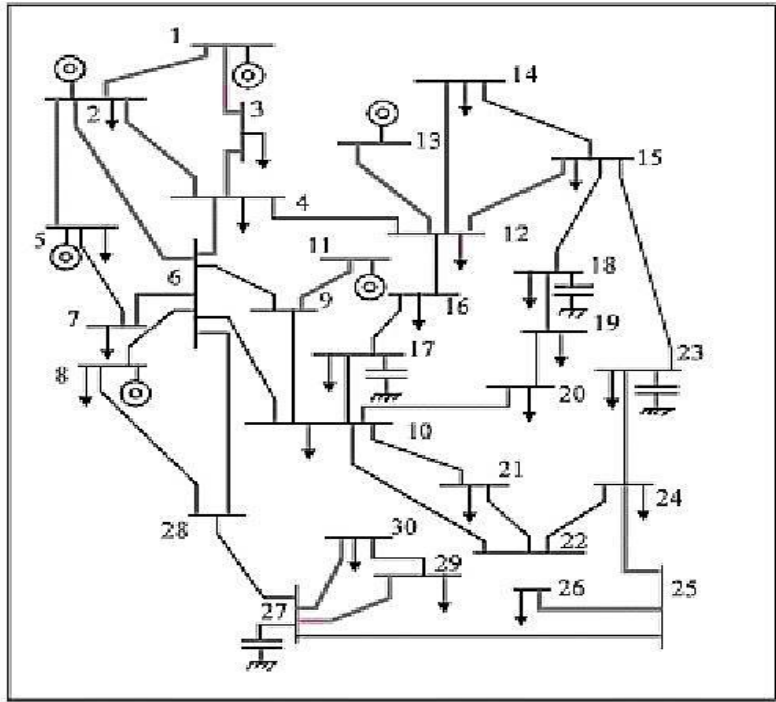

Figure 5. Single line diagram of IEEE-30 Bus System 
Table 2. Comparative analysis for Optimal Dispatch and Total Cost without loss before DG placement

\begin{tabular}{ccccccc}
\hline S.no & Bus No & GA in MW [38] & PSO in MW [39] & ALO in MW & ALO-PSO in MW & ALO-FLC in MW \\
\hline $\mathbf{1}$ & 1 & 137.99 & 143.35 & 142.88 & 178.62 & 186.73 \\
$\mathbf{2}$ & 2 & 64.4 & 51.19 & 51.28 & 41.68 & 37.79 \\
$\mathbf{3}$ & 5 & 15 & 22.98 & 30.03 & 17.53 & 19.67 \\
$\mathbf{4}$ & 8 & 18.32 & 27.69 & 21.66 & 13.14 & 10 \\
$\mathbf{5}$ & 11 & 16.53 & 18.97 & 19.88 & 13.01 & 10.29 \\
$\mathbf{6}$ & 13 & 39.95 & 19.78 & 17.64 & 19.30 & 18.90 \\
\hline \multicolumn{2}{r}{ Total Generation } & 283.4 & 283.4 & 283.4 & 283.4 & 283.4 \\
\multicolumn{2}{r}{ Time } & $2.52 \mathrm{Sec}$ & $2.41 \mathrm{Sec}$ & $2.14 \mathrm{Sec}$ & $2.01 \mathrm{Sec}$ & $1.8 \mathrm{Sec}$ \\
\multicolumn{2}{r}{ Fuel Cost } & $\$ 793.60$ & $\$ 787.77$ & $\$ 799.93$ & $\$ 778.58$ & $\$ 776.74$ \\
\hline
\end{tabular}

Table 3. Comparative analysis for Optimal Dispatch and Total Cost with loss before DG placement

\begin{tabular}{ccccccc}
\hline S.no & Bus No & GA in MW & PSO in MW & ALO in MW & ALO-PSO in MW & ALO-FLC in MW \\
\hline $\mathbf{1}$ & 1 & 140.91 & 146.27 & 184.32 & 183.71 & 197.28 \\
$\mathbf{2}$ & 2 & 67.32 & 54.11 & 47.74 & 55.93 & 49.56 \\
$\mathbf{3}$ & 5 & 17.92 & 25.9 & 20.71 & 18.37 & 18.77 \\
$\mathbf{4}$ & 8 & 21.24 & 30.61 & 14.08 & 10.21 & 10.00 \\
$\mathbf{5}$ & 11 & 19.45 & 21.89 & 17.56 & 12.10 & 11.21 \\
$\mathbf{6}$ & 13 & 42.87 & 22.7 & 16.48 & 20.57 & 14.08 \\
\hline \multicolumn{2}{r}{ Total Generation } & 300.92 & 300.92 & 300.92 & 300.92 & 300.92 \\
\multicolumn{2}{c}{ Time } & $2.65 \mathrm{Sec}$ & $2.45 \mathrm{Sec}$ & $2.15 \mathrm{Sec}$ & $2 \mathrm{Sec}$ & $1.81 \mathrm{Sec}$ \\
\multicolumn{2}{c}{ Fuel Cost } & $\$ 854.59$ & $\$ 848.40$ & $\$ 838.19$ & $\$ 837.60$ & $\$ 833.41$ \\
\hline
\end{tabular}

Table 4. Comparative analysis for Optimal Dispatch and Total Cost with loss after DG [38] placement

\begin{tabular}{ccccccc}
\hline S.no & Bus No & GA in MW & PSO in MW & ALO in MW & ALO-PSO in MW & ALO-FLC in MW \\
\hline $\mathbf{1}$ & 1 & 138.32 & 143.68 & 184.01 & 166.50 & 178.34 \\
$\mathbf{2}$ & 2 & 64.73 & 51.52 & 37.171 & 47.915 & 38.384 \\
$\mathbf{3}$ & 5 & 15.33 & 22.31 & 21.011 & 23.105 & 24.334 \\
$\mathbf{4}$ & 8 & 18.65 & 28.02 & 12.041 & 18.185 & 10.734 \\
$\mathbf{5}$ & 11 & 16.86 & 19.30 & 12.361 & 16.185 & 13.954 \\
$\mathbf{6}$ & 13 & 40.28 & 20.11 & 18.801 & 13.505 & 19.654 \\
\hline \multicolumn{2}{r}{ Total Generation } & 285.4 & 285.4 & 285.4 & 285.4 & 285.4 \\
\multicolumn{2}{c}{ Time } & $2.13 \mathrm{Sec}$ & $1.9 \mathrm{Sec}$ & $2.13 \mathrm{Sec}$ & $1.9 \mathrm{Sec}$ & $1.6 \mathrm{Sec}$ \\
\multicolumn{2}{c}{ Fuel Cost } & $\$ 812.82$ & $\$ 805.47$ & $\$ 812.82$ & $\$ 805.47$ & $\$ 798.40$ \\
\hline
\end{tabular}

Total cost without loss comparison

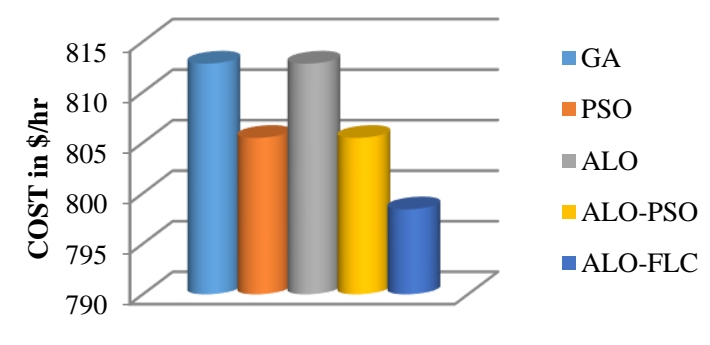

Figure 6. Comparative analysis for Optimal Dispatch and Total Cost without loss before DG placement [39]
Total cost with loss comparison

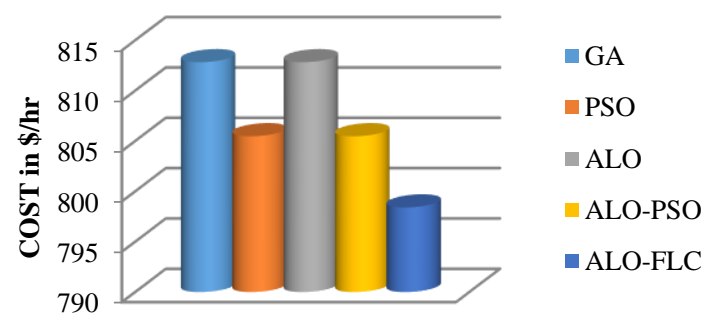

Figure 7. Comparative analysis for Optimal Dispatch and Total Cost with loss before DG placement

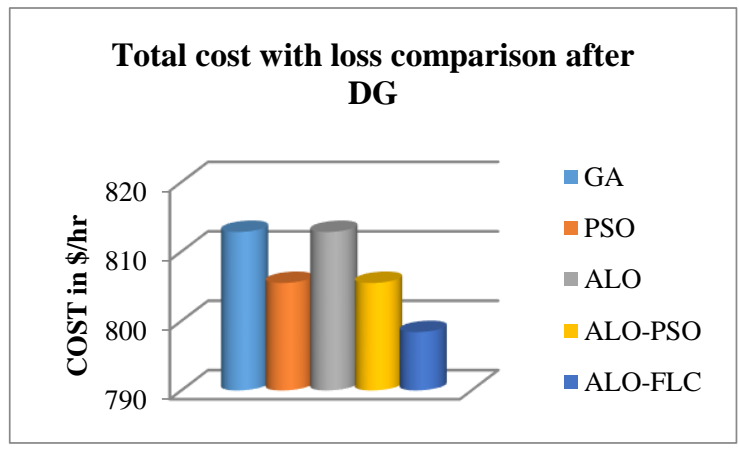

Figure 8. Comparative analysis for Optimal Dispatch and Total Cost with loss after DG placement 


\section{CONCLUSION}

An efficient method is proposed in this research to solve an EPD problem in IEEE-30 bus system. Initially, NR method was considered for optimal load flow issues. In addition, the expressed optimal load flow problems have been fixed while satisfying system inequality and equality constraints. Proposed FLC hybrid technique was verified for its efficiency by initializing the iteration process with a decent principal value and reaches an absolute best value in a smaller number of iterations. Hence, it is decided that the final improvement in cost minimization, scheduling all committed generating units in an optimal way to minimize the total operating cost.

\section{REFERENCES}

[1] Prasanna, T.S., Somasundaram, P. (2008). Fuzzy mutated evolutionary programing based algorithm for combined economic and emission dispatch. IEEE Region 10 Conference, Hyderabad, India, pp. 1-5. https://doi.org/10.1109/TENCON.2008.4766769

[2] Chowdhury, B.H., Saifur, R. (1990). A review of recent advances in economic dispatch. IEEE Transactions on Power Systems, 5(4): 1248-1259. https://doi.org/10.1109/59.99376

[3] Le, K.D., Golden, J.L., Stansbeny, C.J., Vice, R.L., Wood, J.T., Ballance, J., Brown, G., Kamya, J.Y., Nielsen, E.K., Nakajima, H., Ookubo, M., Iyoda, I., Cauley, G.W. (1995). Potential impacts of clean air regulations on system operations. IEEE Transactions on Power Systems, 10(2): 647-653. https://doi.org/10.1109/59.387899

[4] Gent, M.R., Lamont, J.W. (1971). Minimum-emission dispatch. IEEE Transactions on Power Apparatus and Systems, $\quad 90(6)$ : 2650-2660. https://doi.org/10.1109/TPAS.1971.292918

[5] King, T.D., El-Hakwary, M.E., El-Harwary, F. (1995). Optimal environmental dispatching of electric power systems via an improved Hopfield neural network model. IEEE Transactions on Power Systems, 10(3): 1559-1565. https://doi.org/10.1109/59.466488

[6] Song, Y.H., Li, F., Morgan, R., Williams, D. (1995). Environmentally constrained electric power dispatch with genetic algorithms. 1995 IEEE International Coference on Evolutionary Computation, Perth, Australia. https://doi.org/10.1109/ICEC.1995.489112

[7] Sreenivasan, G., Saibabu, C.H., Sivanagaraju, S. (2011). Solution of dynamic economic load dispatch (DELD) problem with valve point loading effects and ramp rate limits using PSO international. Journal of Electrical and Computer Engineering (IJECE), 1(1): 59-70. http://dx.doi.org/10.11591/ijece.v1i1.60

[8] Abu-Mouti, F.S., El-Hawary, M.E. (2011). Heuristic curve-fitted technique for distributed generation optimisation in radial distribution feeder systems. IET Generation, Transmission, Distribution, 5(2): 172-180. http://doi.org/10.1049/iet-gtd.2009.0739

[9] Dasan, S.G.B., Ramalakshmi, S.S., Devi, R.P.K. (2009). Optimal siting and sizing of hybrid distributed generation using EP. International Conference on Power Systems, Kharagpur, India, pp. 1-6. http://doi.org/10.1109/ICPWS.2009.5442761

[10] Mahipal, B., Naik, G.B., Kumar, C.N. (2016). A novel method for determining optimal location and capacity of DG and capacitor in radial network using weightimproved particle swarm optimisation algorithm (WIPSO). An International Journal of Advanced Research in Electrical, Electronics and Instrumentation Engineering, 5(5): 3478-3485. http://doi.org/10.15662/IJAREEIE.2016.0505004

[11] Linh, N.T., Dong, D.X. (2013). Optimal location and size of distributed generation in distribution system by artificial bees colony algorithm. An International Journal of Information and Electronics Engineering, 3(1): 63-67. http://doi.org/10.7763/IJIEE.2013.V3.267

[12] El-Zonkoly, A.M. (2011). Optimal placement of multidistributed generation units including different load models using particle swarm optimization. IET Generation, Transmission, Distribution, 5(7): 760-771. https://doi.org/10.1016/j.swevo.2011.02.003

[13] Samala, R.K., Kotapuri, M.R. (2018). Distributed generation in distribution system for power quality enhancement. International Journal of Engineering \& Technology, $7(4)$ :

24. http://doi.org/10.14419/ijet.v7i4.24.21881

[14] Samala, R.K., Kotapuri, M.R. (2020). Optimal allocation of distributed generations using hybrid technique with fuzzy logic controller radial distribution system. SN Applied Sciences, 191. https://doi.org/10.1007/s42452-020-1957-3

[15] Narayanan, K., Shahbaz, A.S., Manoj, F. (2015). Identification and reduction of impact of islanding using hybrid method with distributed generation. IEEE Power \& Energy Society General Meeting, Denver, USA, pp. 15. http://doi.org/10.1109/PESGM.2015.7286467

[16] Gomez-Gonzalez, M., Lopez, A., Jurado, F. (2012). Optimization of distributed generation systems using a new discrete PSO and OPF. An International Journal of Electric Power Systems Research, 84(1): 174-180. http://doi.org/10.1016/j.epsr.2011.11.016

[17] Samala, R.K., Kotapuri, M.R. (2020) Distributed generation allocation in distribution system using particle swarm optimization based ant-lion optimization. International Journal of Control and Automation, 13(1): 414-426.

[18] Prakash, P., Khatod, D.K. (2016). Optimal sizing and siting techniques for distributed generation in distribution systems: A review. An International Journal of Renewable and Sustainable Energy Reviews, 57: 111130. http://doi.org/10.1016/j.rser.2015.12.099

[19] Mezger, A.J., de Almeida, K.C. (2007). Short term hydro thermal scheduling with bilateral traction via bundle method. International Journal of Electrical Power \& Energy Systems, 29(5): 387-396. https://doi.org/10.1016/j.ijepes.2006.10.002

[20] Ramos, J.L.M., Lora, A.T., Santos, J.R., Exposito, A.G. (2001). Short term hydrothermal coordination based on interior point nonlinear programming and genetic algorithms. IEEE Porto Power Tech. Conference, Porto, Portugal, $\mathrm{pp}$. https://doi.org/10.1109/PTC.2001.964887

[21] Chang, G.W., Aganagic, M., Waight, J.G., Medina, J., Burton, T., Reeves, S. (2001). Experience with mixed integer linear programming based approach on short term hydrothermal scheduling. IEEE Transaction on Power System, 16(4): https://doi.org/10.1109/59.962421 
[22] Santos, A., da Costa, G.R. (1989). Optimal power flow by Newtons method applied to an augmented Lagrangian function. IEEE Proceedings Generation, Transmission \& Distribution, 142(1): 33-36. https://doi.org/10.1049/ipgtd: 19951586

[23] Lin, W.M., Cheng, F.S., Tsay, M.T. (2002). An improved Tabu search for economic dispatch with multiple minima. IEEE Transaction on Power System, 17(2): 108-112. https://doi.org/10.1109/59.982200

[24] Bouktir, T., Slimani, L., Belkacemi, M. (2004). A genetic algorithm for solving for the optimal power flow problem. Leonardo Journal of Sciences, 4: 44-58.

[25] Allaoua, B., Laoufi, A. (2009). Optimal power flow solution Unsing ant manners for electrical network. Advance in Electrical and Computer Engineering, 9: 3440. https://doi.org/10.4316/AECE.2009.01006

[26] Kennedy, J., Eberhart, R. (1995). Particle swarm optimization. IEEE International Conference on Neural Networks, Perth, Australia, 4: 1942-1948. https://doi.org/10.1109/ICNN.1995.488968

[27] Samala, R.K., Kotapuri, M.R. (2018). Optimal DG sizing and siting in radial system using hybridization of GSA and firefly algorithms. Modelling, Measurement and Control A, 91(2): 77-82. https://doi.org/10.18280/mmca.910208

[28] Yalcinoz, T., Altun, H., Uzam, M. (2001). Economic dispatch solution using genetic algorithm based on arithmetic crossover. IEEE Porto Power Tech. Conference, Porto, Portugal, p. 4. https://doi.org/10.1109/PTC.2001.964734

[29] Nanda, J., Naryan, R.B. (2001). Application of genetic algorithm to economic load dispatch with line flow constraints. International Journal of Electrical Power \& Energy Systems, 24(9): 723-729. https://doi.org/10.1016/S0142-0615(02)00002-9

[30] Roa-Sepulveda, C.A., Herrrera, M., Pavez-Lazo, B., Knight, U.G., Coonick, A.H. (2003). Economic dispatch using fuzzy decision trees. Electric Power System Research, 66(2): 115-122. https://doi.org/10.1016/S0378-7796(03)00020-8

[31] Bouktir, T., Mahdad, B., Bouktir, T., Srairi, K. (2003). Fuzzy controlled genetic algorithm for environmental/economic dispatch with shunt FACTS devices. IEEE/PES Transmission and Distribution Conference and Exposition, Chicago, USA, pp. 1-8. https://doi.org/10.1109/TDC.2008.4517069

[32] Benasla, L., Belmadani, A., Mostefa, R. (2008). HookeJeeves method applied to a new economic dispatch problem formulation. Journal of Information Science and Engineering, 24(3): 907-917.

[33] Sushil, K., Naresh, R. (2008). Nonconvex economic load dispatch using an efficient real coded genetic algorithm. Applied Soft Computing, 9(1): 321-329. https://doi.org/10.1016/j.asoc.2008.04.009

[34] Jagabondhu, H., Avinash, S. (2009). Application of soft computing methods for economic dispatch in power systems. International Journal of Electrical Power and Energy Systems, 538-543.

[35] Samala, R.K., Kotapuri, M.R. (2017). Optimal allocation of distributed generations using hybrid technique with fuzzy logic controller radial distribution system. SN Applied $\quad$ Sciences, 191. https://doi.org/10.1007/s42452-020-1957-3

[36] Samala, R.K., Kotapuri, M.R. (2017). Multi distributed generation placement using ant-lion optimization. European Journal of Electrical Engineering, 5(6): 253267. https://doi.org/ 10.3166/ejee.19.256-267

[37] Alsac, O., Stott, B. (1974). Optimal load flow with steady-state security. IEEE Transactions on Power Apparatus and Systems, 93(3): 745-751. https://doi.org/10.1109/TPAS.1974.293972

[38] Samala, R.K., Kotapuri, M.R. (2018). Power loss reduction using distributed generation. Modelling, Measurement and Control A, 91(3): 104-113. https://doi.org/10.18280/mmc_a.910302

[39] Samala, R.K., Kotapuri, M.R. (2018). Power enhancement by integrating distributed generation in distribution system. International Journal of Engineering \& Technology, 7(4.24): 167-171. https://doi.org/10.14419/ijet.v7i4.24.21881

\section{NOMENCLATURE}

$\begin{array}{ll}\mathrm{F}_{\text {cost }} & \text { cost Function } \\ \mathrm{a}_{\mathrm{i}}, \mathrm{b}_{\mathrm{i}}, \mathrm{c}_{\mathrm{i}} & \text { cost coefficients } \\ \mathrm{P}_{\mathrm{i}} & \text { real output } \\ \mathrm{N}_{\mathrm{g}} & \text { total number of generators } \\ \text { cumsum } & \text { total sum } \\ \mathrm{n} & \text { total ants } \\ \mathrm{t} & \text { random walk } \\ \mathrm{r}(\mathrm{t}) & \text { random generation }\end{array}$

\section{Subscripts}

$\begin{array}{ll}\text { EPD } & \text { Economic Power Dispatch } \\ \text { FLC } & \text { Fuzzy Logic Controller } \\ \text { ALO } & \text { Ant-Lion Optimization } \\ \text { LSF } & \text { Loss sensitivity Factor } \\ \text { S } & \text { Short } \\ \text { M } & \text { Medium } \\ \text { L } & \text { Large }\end{array}$

